\title{
Divergence Studies for Different Horticultural Traits in Cucumber (Cucumis sativus L.)
}

\author{
Shweta Sharma ${ }^{1 *}$, Ramesh Kumar ${ }^{1}$, H.R. Sharma ${ }^{1}$, Ajit Sharma ${ }^{2}$ and Nidhish Gautam ${ }^{1}$ \\ ${ }^{1}$ Department of Vegetable Science, ${ }^{2}$ Department of Basic Science, Dr YS Parmar University of \\ Horticulture and Forestry Nauni, Solan - 173230 (HP), India \\ *Corresponding author
}

\begin{tabular}{|l|}
\hline K e y w or d s \\
Cucumber, \\
Genotypes, Genetic \\
divergence, Cluster
\end{tabular}

\section{Introduction}

Cucumber (Cucumis sativus L.) is one of the most important cucurbitaceous vegetable crops grown extensively in tropical and subtropical parts of the country. It is considered as $4^{\text {th }}$ most important vegetable crop after tomato, cabbage and onion. Cucumber is a thermophilic and frost susceptible species growing best at a temperature above $20^{\circ} \mathrm{C}$. It is grown for its tender fruits, which are consumed either raw as salad, cooked as vegetable or as pickling cucumber in its immature stage. It is a rich source of vitamin $\mathrm{B}$ and $\mathrm{C}$, carbohydrates, $\mathrm{Ca}$ and $\mathrm{P}$. Cucumber is thought to be indigenous to India. India is endowed with the wealth of cucumber germplasm, comprising of both wild and cultivated forms. Due to continuous cultivation of this cross-pollinated crop large variation has occurred for fruit and vegetative characters (Sharma et al., 2017). The success of any breeding program depends to a large extent on the amount of genetic variability 
present in the population (Afangideh and Uyoh, 2007). Suitable breeding strategy can be formulated for improvement of cucumber based on the magnitude of parameters of variability. Therefore, the present study has been undertaken to estimate the extent of variability and genetic divergence in thirty genotypes of cucumber.

Assessment of genetic diversity could be suitable in crop breeding for diverse applications such as identifying diverse parental genotypes. Genetic diversity is the amount of heritable variability between varieties or populations of organisms. Variability occurs from differences in DNA sequences, biochemical characteristics like protein structure or isoenzyme properties, physiological properties like growth rate and morphological characters. Substantial effort has been directed towards collecting, preserving and evaluating genetic variability in crops (Golabadi et al., 2012).

\section{Materials and Methods}

The present study was conducted at the Experimental farm of the Department of Vegetable Science is located at an altitude of 1276 metres above mean sea level at a longitude of $77^{\circ} 11^{\prime} 30^{\prime \prime} \mathrm{E}$ and a latitude of $30^{\circ}$ $52^{\prime} 30^{\prime \prime} \mathrm{N}$ and is $1260 \mathrm{~m}$ above mean sea level and represents the mid-hill zone of Himachal Pradesh. The experimental material comprised of thirty genotypes including check cultivar K75 collected from different exotic and indigenous germplasm of cucumber (Table 1). The experiment was laid out in Randomized Complete Block Design with three replications of each genotype. Seeds were directly sown in the field in the month of June, 2016. Three to four seeds per basin were sown at a spacing of $100 \times 50 \mathrm{~cm}$ in a plot had size of $3.0 \times 2.0 \mathrm{~m}^{2}$, accommodating 12 plants per plot. After the emergence of seedlings, only one healthy plant per hill was retained. The standard cultural practices as recommended in the Package of Practices for Vegetable Crops, were followed to ensure a healthy crop stand (Anonymous, 2014).

The observations were recorded from five randomly selected plants in each replication for all characters except for fruit characters for which observations were recorded on ten randomly selected fruits per replication. Colour of fruits was observed visually after harvesting with the help of colour chart of Royal Horticultural Society, London. Seed germination of each genotype was tested in accordance with ISTA (Anonymous, 1985) and seed vigour index-I and II were calculated as per the formula given by Abdul-Baki and Anderson (1973), where, seed vigour index I = seed germination percentage $\times$ seedling length (cm) and seed vigour index II $=$ seed germination percentage $\times$ seedling dry weight $(\mathrm{mg})$. The disease severity of angular leaf spot was recorded on modified 0-9 scale of Woltman et al., (2009) where absence of disease symptom was taken as 0 and leaf damage of $87-100 \%$ was scored 9. Severity of anthracnose and powdery mildew was recorded on 0-5 scale of Akem and Jovicich (2011). Here, absence of disease symptom was scored as 0 and leaf necrosis between 75$100 \%$ scored 5 . For all the three diseases, the observations were recorded 65 days after sowing from ten leaves in each plant, five such vines in each replication of each genotype. Disease severity percentage was calculated by adopting the formula of McKinney (1923).

\section{Statistical analysis}

The collected data were subjected to Analysis of Variance (ANOVA) using OP-STAT software available from HAU, Hisar and presented in table 2 and genetic divergence (D) was worked out according to Mahalanobis (1936) using SPAR-1 software available from 
IASRI, New Delhi. A dendrogram was generated using Wards method and Euclidan distance as a measure of similarity with the help of SPSS software version 16 and is presented in Figure 1.

\section{Results and Discussion}

The analysis of variance revealed highly significant differences among the genotypes for all the characters studied, indicating the existence of wide genetic divergence among them. Information on genetic diversity was also used to identify promising diverse genotypes, which may further be used in breeding programmes.

On the basis of performance of various traits, the clustering pattern of 30 diverse genotypes of cucumber has been presented in the table 3 . All the genotypes were grouped into 5 clusters. Maximum number of genotypes were accommodated in cluster II (12) followed by cluster IV (7), cluster I and III (4) and cluster V (3). Genotypes from same centre of origin were placed in separate clusters, indicated wide genetic diversity among them. This may be due to frequent exchange of germplasm between different geographical regions.

Average inter and intra cluster divergence $\left(\mathrm{D}^{2}\right)$ values are presented in the table 4 . The diagonal figures in the table represent the intra cluster distances. The intra cluster distance was maximum in cluster IV (3.469) and minimum in cluster III (1.776).

Whereas, highest (6.221) inter cluster distance was recorded between cluster III and IV and lowest (2.502) was observed between cluster I and II suggesting wide diversity between the groups. The crosses made between the genotypes from the above clusters may give transgressive segregants.

Fig.1 Dendrogram using hierarchical cluster analysis

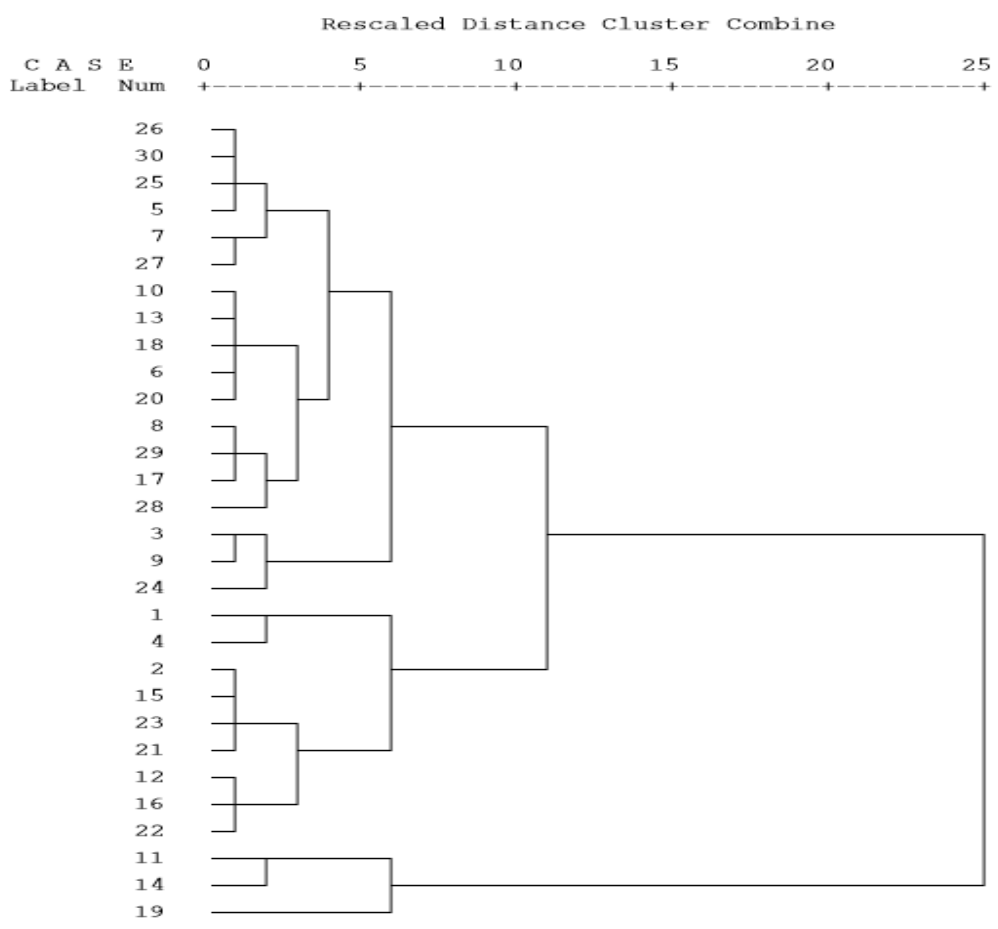


Table.1 List of cucumber genotypes studied along with their sources

\begin{tabular}{|c|c|}
\hline Genotype & Source \\
\hline $\begin{array}{l}\text { CGN-19533, CGN-20269, CGN-20515, } \\
\text { CGN-20827, CGN-20930, CGN-20953, } \\
\text { CGN-20969, CGN-21585, CGN-22930 }\end{array}$ & $\begin{array}{l}\text { Centre for Crop Genetic Resources, the } \\
\text { Netherlands }\end{array}$ \\
\hline PI-426170, PI-5754, PI-618894 & $\begin{array}{l}\text { North Central Regional Plant Introduction } \\
\text { Station, USA }\end{array}$ \\
\hline $\begin{array}{l}\text { UHF-CUC-4, UHF-CUC-5, UHF-CUC-6, } \\
\text { UHF-CUC-7, UHF-CUC-8, UHF-CUC-9, } \\
\text { UHF-CUC-10, UHF-CUC-11, UHF-CUC- } \\
\text { 12, UHF-CUC-13, UHF-CUC-14, UHF- } \\
\text { CUC-15, UHF-CUC-16, UHF-CUC-17, } \\
\text { UHF-CUC-18, UHF-CUC-19, Poinsette, K- } \\
75 \text { (Check) }\end{array}$ & $\begin{array}{l}\text { Department of Vegetable Science, UHF, Solan, } \\
\text { HP }\end{array}$ \\
\hline
\end{tabular}

Table.2 Analysis of variance for various horticultural traits in cucumber

\begin{tabular}{|l|}
\hline $\begin{array}{l}\text { Source } \\
\text { Character }\end{array}$ \\
\hline Df \\
\hline Node number bearing first female \\
\hline Days to marketable maturity \\
\hline Number of marketable fruits/plant \\
\hline Harvest duration (days) \\
\hline Fruit length(cm) \\
\hline Fruit breadth (cm) \\
\hline Average fruit weight (g) \\
\hline Yield/ plot (kg) \\
\hline Yield /hectare (q) [converted] \\
\hline TSS ( $B$ B) \\
\hline Seed germination (\%) \\
\hline Seed vigour index-I \\
\hline Seed vigour index-II \\
\hline Powdery mildew (\%) \\
\hline Anthracnose (\%) \\
\hline Angular leaf spot (\%) \\
\hline
\end{tabular}

\begin{tabular}{|c|c|c|c|}
\hline \multicolumn{4}{|c|}{ Mean Sum of Squares } \\
\hline Replications & Genotypes & Errors & Total \\
\hline 2 & & & \\
\hline 0.28 & $5.65^{*}$ & 0.37 & 69 \\
\hline 10.07 & $164.42^{*}$ & 3.97 & 178.46 \\
\hline 0.09 & $4.18^{*}$ & 0.18 & 4.45 \\
\hline 0.62 & $54.22^{*}$ & 3.02 & 57.86 \\
\hline 1.28 & $16.38^{*}$ & 1.25 & 18.91 \\
\hline 0.26 & $0.65^{*}$ & 0.10 & 1.01 \\
\hline $2279.71^{*}$ & $4410.97^{*}$ & 492.96 & 7183.64 \\
\hline 0.19 & $55.39^{*}$ & 1.30 & 56.88 \\
\hline 28.40 & $8654.93^{*}$ & 202.72 & 8886.05 \\
\hline $0.08^{*}$ & $0.10^{*}$ & 0.02 & 0.20 \\
\hline 20.81 & $120.52^{*}$ & 15.33 & 156.66 \\
\hline 2143.60 & $408017.42^{*}$ & 19125.65 & 429286. \\
\hline 68815.70 & $216697.65^{*}$ & 30073.26 & 315586. \\
\hline $12.18^{*}$ & $27.75^{*}$ & 2.81 & 42.74 \\
\hline 4.36 & $76.26^{*}$ & 3.25 & 83.87 \\
\hline 1.11 & $139.35^{*}$ & 3.08 & 143.54 \\
\hline
\end{tabular}

*Significant at $5 \%$ level of significance 
Table.3 Clustering pattern of 30 genotypes of cucumber on the basis of genetic divergence

\begin{tabular}{|c|c|c|}
\hline Cluster & $\begin{array}{l}\text { Number of } \\
\text { Genotypes }\end{array}$ & Genotypes along with their sources \\
\hline I & 4 & $\begin{array}{l}\text { CGN-20269 (the Netherlands), CGN-22930 (the Netherlands), UHF- } \\
\text { CUC-9 (Solan), Poinsette (Solan) }\end{array}$ \\
\hline II & 12 & $\begin{array}{l}\text { CGN-20515 (the Netherlands), CGN-20827 (the Netherlands), CGN- } \\
20930 \text { (the Netherlands), CGN-20953 (the Netherlands), CGN-20969 } \\
\text { (the Netherlands), CGN-21585 (the Netherlands), UHF-CUC-4 } \\
\text { (Solan), UHF-CUC-7 (Solan), UHF-CUC-11(Solan), PI-426170 } \\
\text { (USA), PI-5754 (USA), K-75 (Solan) }\end{array}$ \\
\hline III & 4 & $\begin{array}{l}\text { UHF-CUC-8 (Solan), UHF-CUC-15 (Solan), UHF-CUC-16 (Solan), } \\
\text { UHF-CUC-17 (Solan) }\end{array}$ \\
\hline$\overline{\text { IV }}$ & 7 & $\begin{array}{l}\text { CGN-19533 (the Netherlands), UHF-CUC-5 (Solan), UHF-CUC-10 } \\
\text { (Solan), UHF-CUC-12 (Solan), UHF-CUC-13 (Solan), UHF-CUC-18 } \\
\text { (Solan), UHF-CUC-19 (Solan) }\end{array}$ \\
\hline $\mathbf{V}$ & 3 & UHF-CUC-6 (Solan), UHF-CUC-14 (Solan), PI-618894 (USA) \\
\hline
\end{tabular}

Table.4 Average intra and inter cluster distance $\left(\mathrm{D}^{2}\right)$

\begin{tabular}{|l|l|l|l|l|l|}
\hline Cluster & I & II & III & IV & V \\
\hline I & $\underline{\mathbf{2 . 2 9 9}}$ & & & & \\
\hline II & 2.502 & $\underline{\mathbf{2 . 3 8 0}}$ & & & \\
\hline III & 3.928 & 5.275 & $\underline{\mathbf{1 . 7 7 6}}$ & & \\
\hline IV & 4.546 & 3.206 & 6.221 & $\underline{\mathbf{3 . 4 6 9}}$ & $\mathbf{3 . 1 0 9}$ \\
\hline V & 4.314 & 3.493 & 6.036 & $\underline{\mathbf{3 . 1 0}}$ & \\
\hline
\end{tabular}

Table.5 Cluster means for different characters among 30 genotypes of cucumber

\begin{tabular}{|l|}
\hline Characters \\
\hline Node number bearing $1^{\text {st }}$ female flower \\
\hline Number of marketable fruits / plant \\
\hline Fruit length (cm) \\
\hline Fruit breadth (cm) \\
\hline Average fruit weight (g) \\
\hline Days to marketable maturity \\
\hline Harvest duration (days) \\
\hline TSS ( $\mathrm{B})$ \\
\hline Seed germination (\%) \\
\hline Seed vigour index-I \\
\hline Seed vigour index-II \\
\hline Severity of powdery mildew (\%) \\
\hline Severity of anthracnose (\%) \\
\hline Severity of angular leaf spot (\%) \\
\hline Yield/plot (kg) \\
\hline
\end{tabular}

\begin{tabular}{|l|l|l|l|l|}
\hline I & II & III & IV & V \\
\hline 4.53 & 5.18 & 3.75 & 6.41 & 4.89 \\
\hline 7.84 & 6.83 & 8.23 & 5.62 & 6.25 \\
\hline 15.06 & 16.41 & 18.45 & 15.75 & 21.42 \\
\hline 4.71 & 4.87 & 5.09 & 5.50 & 4.85 \\
\hline 251.98 & 267.00 & 314.21 & 274.35 & 224.31 \\
\hline 49.43 & 48.88 & 47.43 & 60.23 & 52.02 \\
\hline 23.25 & 19.68 & 28.83 & 17.90 & 18.09 \\
\hline 2.99 & 2.48 & 3.04 & 2.91 & 2.58 \\
\hline 89.33 & 83.94 & 99.42 & 87.57 & 86.44 \\
\hline 2145.92 & 1752.05 & 2431.21 & 2042.55 & 1846.58 \\
\hline 1119.22 & 1094.03 & 1431.76 & 1312.41 & 1348.14 \\
\hline 14.27 & 13.19 & 9.72 & 10.70 & 14.52 \\
\hline 9.36 & 13.23 & 9.06 & 17.72 & 7.48 \\
\hline 9.82 & 14.81 & 5.68 & 18.59 & 6.87 \\
\hline 22.60 & 20.03 & 26.93 & 16.87 & 19.11 \\
\hline
\end{tabular}




\section{Int.J.Curr.Microbiol.App.Sci (2018) 7(2): 1733-1741}

Table.6 Mean performance of cucumber genotypes for different horticultural traits

\begin{tabular}{|c|c|c|c|c|c|c|c|c|c|c|c|c|c|c|c|c|c|}
\hline Genotype & NNBFF & DMM & NMF & HD & FL & FB & AFW & TSS & FC & SG $*$ & SVI-I & SVI-II & $\mathrm{SPM}^{*}$ & $\mathrm{SA}^{*}$ & $\begin{array}{c}\text { SALS } \\
*\end{array}$ & YPP & YPH \\
\hline CGN-19533 & 6.83 & 65.47 & 6.07 & 19.33 & 15.50 & 4.77 & 252.94 & 3.08 & $\begin{array}{l}\text { LG (G } \\
143 \text { B) }\end{array}$ & $\begin{array}{r}96.67 \\
(9.89)\end{array}$ & 1919.31 & 1735.93 & $\begin{array}{l}13.00 \\
(3.73)\end{array}$ & $\begin{array}{l}17.07 \\
(4.24)\end{array}$ & $\begin{array}{l}13.60 \\
(3.81)\end{array}$ & 17.48 & 218.46 \\
\hline CGN-20269 & 3.26 & 44.33 & 7.55 & 19.80 & 15.67 & 4.90 & 247.67 & 2.80 & $\begin{array}{l}\text { LG (G } \\
147 \mathrm{~A})\end{array}$ & $\begin{array}{l}85.67 \\
(9.31)\end{array}$ & 2243.79 & 1207.41 & $\begin{array}{l}18.73 \\
(4.44)\end{array}$ & $\begin{array}{c}9.50 \\
(3.24)\end{array}$ & $\begin{array}{l}11.27 \\
(3.49)\end{array}$ & 21.48 & 268.50 \\
\hline CGN-20515 & 5.33 & 44.13 & 8.09 & 18.40 & 16.13 & 4.65 & 273.50 & 2.79 & $\begin{array}{l}\text { LG (G } \\
146 \text { B) }\end{array}$ & $\begin{array}{l}82.33 \\
(9.13)\end{array}$ & 1898.55 & 903.85 & $\begin{array}{l}14.47 \\
(3.92)\end{array}$ & $\begin{array}{l}18.83 \\
(4.45)\end{array}$ & $\begin{array}{l}17.60 \\
(4.31)\end{array}$ & 23.72 & 296.54 \\
\hline CGN-20827 & 3.89 & 42.47 & 7.27 & 18.33 & 15.47 & 5.41 & 255.00 & 2.55 & $\begin{array}{l}\text { LG (G } \\
144 \text { B) }\end{array}$ & $\begin{array}{l}81.33 \\
(9.08)\end{array}$ & 1850.29 & 1473.47 & $\begin{array}{l}13.33 \\
(3.78)\end{array}$ & $\begin{array}{l}11.73 \\
(3.57)\end{array}$ & $\begin{array}{l}11.00 \\
(3.46)\end{array}$ & 24.40 & 305.00 \\
\hline CGN-20930 & 6.83 & 45.27 & 6.98 & 19.00 & 15.28 & 5.32 & 308.47 & 2.71 & $\begin{array}{c}\mathrm{G}(\mathrm{G} \\
138 \mathrm{~A})\end{array}$ & $\begin{array}{l}85.00 \\
(9.28)\end{array}$ & 1840.64 & 1072.10 & $\begin{array}{l}13.40 \\
(3.79)\end{array}$ & $\begin{array}{l}11.47 \\
(3.51)\end{array}$ & $\begin{array}{l}16.27 \\
(4.15)\end{array}$ & 21.43 & 267.88 \\
\hline CGN-20953 & 5.83 & 60.87 & 7.85 & 18.67 & 15.67 & 4.58 & 285.17 & 2.71 & $\begin{array}{c}\mathrm{G}(\mathrm{G} \\
141 \mathrm{~B})\end{array}$ & $\begin{array}{l}82.67 \\
(9.15)\end{array}$ & 1457.82 & 1165.84 & $\begin{array}{l}12.83 \\
(3.72)\end{array}$ & $\begin{array}{c}9.53 \\
(3.25)\end{array}$ & $\begin{array}{l}17.27 \\
(4.27)\end{array}$ & 25.31 & 316.42 \\
\hline CGN-20969 & 5.67 & 44.93 & 6.44 & 18.40 & 15.47 & 4.47 & 283.33 & 3.07 & $\begin{array}{c}\mathrm{G}(\mathrm{G} \\
139 \mathrm{~B})\end{array}$ & $\begin{array}{l}83.67 \\
(9.20)\end{array}$ & 2017.63 & 1119.94 & $\begin{array}{l}13.27 \\
(3.77)\end{array}$ & $\begin{array}{l}11.70 \\
(3.56)\end{array}$ & $\begin{array}{l}11.00 \\
(3.46)\end{array}$ & 20.57 & 257.13 \\
\hline CGN-21585 & 5.01 & 47.13 & 6.06 & 19.33 & 16.48 & 5.34 & 263.33 & 3.19 & $\begin{array}{l}\text { LG (G } \\
152 \text { B) }\end{array}$ & $\begin{array}{l}82.33 \\
(9.13)\end{array}$ & 1535.27 & 1009.87 & $\begin{array}{l}10.60 \\
(3.40)\end{array}$ & $\begin{array}{l}12.77 \\
(3.70)\end{array}$ & $\begin{array}{l}19.80 \\
(4.56)\end{array}$ & 15.89 & 198.67 \\
\hline CGN-22930 & 5.50 & 45.33 & 7.87 & 20.47 & 13.10 & 5.00 & 264.05 & 3.07 & $\begin{array}{l}\text { LG (G } \\
152 \mathrm{~A})\end{array}$ & $\begin{array}{l}93.00 \\
(9.69)\end{array}$ & 2052.70 & 823.40 & $\begin{array}{l}17.17 \\
(4.26)\end{array}$ & $\begin{array}{l}10.93 \\
(3.45)\end{array}$ & $\begin{array}{l}12.33 \\
(3.64)\end{array}$ & 22.96 & 287.04 \\
\hline UHF-CUC-4 & 3.44 & 50.47 & 4.54 & 23.33 & 16.80 & 4.57 & 263.33 & 2.79 & $\begin{array}{c}\mathrm{G}(\mathrm{G} \\
137 \mathrm{~A})\end{array}$ & $\begin{array}{l}84.33 \\
(9.24)\end{array}$ & 1642.11 & 1240.89 & $\begin{array}{l}15.63 \\
(4.08)\end{array}$ & $\begin{array}{l}12.53 \\
(3.67)\end{array}$ & $\begin{array}{l}17.33 \\
(4.28)\end{array}$ & 12.38 & 154.79 \\
\hline UHF-CUC-5 & 5.83 & 62.13 & 6.73 & 16.80 & 18.47 & 5.91 & 279.33 & 2.72 & $\begin{array}{l}\text { LG (G } \\
152 \text { B) }\end{array}$ & $\begin{array}{l}83.67 \\
(9.21)\end{array}$ & 2580.43 & 1663.84 & $\begin{array}{c}6.33 \\
(2.69)\end{array}$ & $\begin{array}{l}23.00 \\
(4.89)\end{array}$ & $\begin{array}{l}15.80 \\
(4.09)\end{array}$ & 20.68 & 258.54 \\
\hline UHF-CUC-6 & 3.33 & 51.67 & 6.63 & 16.67 & 19.00 & 4.68 & 256.25 & 2.68 & $\begin{array}{c}\mathrm{G}(\mathrm{G} \\
139 \mathrm{~A})\end{array}$ & $\begin{array}{l}95.33 \\
(9.82)\end{array}$ & 2180.60 & 1601.36 & $\begin{array}{l}15.00 \\
(4.00)\end{array}$ & $\begin{array}{c}8.17 \\
(3.02)\end{array}$ & $\begin{array}{c}4.40 \\
(2.32)\end{array}$ & 18.52 & 231.50 \\
\hline UHF-CUC-7 & 4.33 & 45.33 & 6.26 & 16.33 & 17.47 & 5.13 & 276.67 & 2.93 & $\begin{array}{c}\mathrm{G}(\mathrm{G} \\
137 \mathrm{~A})\end{array}$ & $\begin{array}{l}89.67 \\
(9.53)\end{array}$ & 1602.75 & 1167.33 & $\begin{array}{l}14.47 \\
(3.92)\end{array}$ & $\begin{array}{l}13.97 \\
(3.87)\end{array}$ & $\begin{array}{c}6.33 \\
(2.70)\end{array}$ & 20.34 & 254.25 \\
\hline UHF-CUC-8 & 4.83 & 45.73 & 8.08 & 27.20 & 16.58 & 4.93 & 266.67 & 3.11 & $\begin{array}{c}\mathrm{G}(\mathrm{G} \\
137 \mathrm{~B})\end{array}$ & $\begin{array}{c}99.33 \\
(10.02)\end{array}$ & 2800.87 & 1720.33 & $\begin{array}{l}11.43 \\
\text { (3.52) }\end{array}$ & $\begin{array}{c}8.90 \\
(3.14)\end{array}$ & $\begin{array}{c}5.53 \\
(2.54)\end{array}$ & 25.44 & 317.96 \\
\hline UHF-CUC-9 & 4.50 & 46.07 & 8.16 & 28.40 & 15.90 & 4.59 & 238.33 & 3.07 & $\begin{array}{c}\mathrm{G}(\mathrm{G} \\
143 \mathrm{~A})\end{array}$ & $\begin{array}{l}84.67 \\
(9.26)\end{array}$ & 2208.91 & 1285.72 & $\begin{array}{c}9.50 \\
(3.24)\end{array}$ & $\begin{array}{c}8.10 \\
(3.01)\end{array}$ & $\begin{array}{c}6.67 \\
(2.76)\end{array}$ & 22.50 & 281.21 \\
\hline
\end{tabular}




\begin{tabular}{|c|c|c|c|c|c|c|c|c|c|c|c|c|c|c|c|c|c|}
\hline UHF-CUC-10 & 9.67 & 53.33 & 5.34 & 18.07 & 17.17 & 5.06 & 293.33 & 3.04 & $\begin{array}{c}\mathrm{G}(\mathrm{G} \\
142 \mathrm{~A})\end{array}$ & $\begin{array}{l}94.00 \\
(9.75)\end{array}$ & 2202.76 & 1443.88 & $\begin{array}{l}12.93 \\
(3.73)\end{array}$ & $\begin{array}{l}27.07 \\
(5.30)\end{array}$ & $\begin{array}{c}9.53 \\
(3.25)\end{array}$ & 18.36 & 229.46 \\
\hline UHF-CUC-11 & 5.22 & 57.00 & 5.94 & 23.20 & 15.08 & 5.01 & 253.75 & 2.71 & $\begin{array}{c}\mathrm{G}(\mathrm{G} \\
143 \mathrm{~B})\end{array}$ & $\begin{array}{l}83.33 \\
(9.19)\end{array}$ & 1690.65 & 846.92 & $\begin{array}{l}13.47 \\
(3.79)\end{array}$ & $\begin{array}{l}17.50 \\
(4.29)\end{array}$ & $\begin{array}{l}12.67 \\
(3.69)\end{array}$ & 19.92 & 248.96 \\
\hline UHF-CUC-12 & 5.33 & 50.67 & 4.04 & 15.33 & 14.67 & 6.00 & 328.92 & 2.71 & $\begin{array}{l}\mathrm{LG}(\mathrm{G} \\
152 \mathrm{~A})\end{array}$ & $\begin{array}{l}84.33 \\
(9.24)\end{array}$ & 1695.51 & 1293.53 & $\begin{array}{l}13.67 \\
(3.82)\end{array}$ & $\begin{array}{l}14.73 \\
(3.96)\end{array}$ & $\begin{array}{l}29.00 \\
(5.48)\end{array}$ & 11.43 & 142.84 \\
\hline UHF-CUC-13 & 5.33 & 62.00 & 5.13 & 20.80 & 15.97 & 5.51 & 255.33 & 2.87 & $\begin{array}{l}\text { LG (G } \\
144 \mathrm{~A})\end{array}$ & $\begin{array}{l}84.00 \\
(9.22)\end{array}$ & 2829.07 & 1264.83 & $\begin{array}{c}7.07 \\
(2.83)\end{array}$ & $\begin{array}{l}15.97 \\
(4.12)\end{array}$ & $\begin{array}{l}23.27 \\
(4.93)\end{array}$ & 20.85 & 260.63 \\
\hline UHF-CUC-14 & 6.33 & 57.73 & 6.33 & 17.60 & 19.83 & 5.37 & 141.50 & 2.53 & $\begin{array}{l}\text { LG (G } \\
149 \mathrm{C})\end{array}$ & $\begin{array}{l}80.67 \\
(9.04)\end{array}$ & 1557.80 & 1211.35 & $\begin{array}{l}18.83 \\
(4.45)\end{array}$ & $\begin{array}{l}10.93 \\
(3.45)\end{array}$ & $\begin{array}{l}12.20 \\
(3.62)\end{array}$ & 19.68 & 246.00 \\
\hline UHF-CUC-15 & 3.67 & 45.60 & 8.15 & 29.20 & 18.88 & 4.79 & 307.08 & 3.03 & $\begin{array}{c}\mathrm{G}(\mathrm{G} \\
143 \mathrm{~A})\end{array}$ & $\begin{array}{l}100.00 \\
(10.05)\end{array}$ & 2361.33 & 1155.00 & $\begin{array}{c}8.80 \\
(3.13)\end{array}$ & $\begin{array}{c}8.67 \\
(3.10)\end{array}$ & $\begin{array}{c}6.07 \\
(2.64)\end{array}$ & 25.64 & 320.50 \\
\hline UHF-CUC-16 & 2.83 & 53.67 & 8.21 & 29.93 & 19.12 & 5.66 & 341.67 & 3.01 & $\begin{array}{c}\mathrm{G}(\mathrm{G} \\
141 \mathrm{~B})\end{array}$ & $\begin{array}{c}99.33 \\
(10.02)\end{array}$ & 2327.61 & 1576.24 & $\begin{array}{c}8.50 \\
(3.08)\end{array}$ & $\begin{array}{c}9.50 \\
(3.23)\end{array}$ & $\begin{array}{c}4.73 \\
(2.39)\end{array}$ & 27.94 & 349.21 \\
\hline UHF-CUC-17 & 3.67 & 44.73 & 8.49 & 29.00 & 19.23 & 5.00 & 341.44 & 3.02 & $\begin{array}{c}\mathrm{G}(\mathrm{G} \\
141 \mathrm{~A})\end{array}$ & $\begin{array}{l}99.00 \\
(10.00)\end{array}$ & 2235.03 & 1275.46 & $\begin{array}{l}10.13 \\
(3.34)\end{array}$ & $\begin{array}{c}9.17 \\
(3.19)\end{array}$ & $\begin{array}{c}6.40 \\
(2.69)\end{array}$ & 28.71 & 358.92 \\
\hline PI-426170 & 5.33 & 46.67 & 7.88 & 18.60 & 18.17 & 4.55 & 251.50 & 2.76 & $\begin{array}{c}\mathrm{G}(\mathrm{G} \\
139 \mathrm{~A})\end{array}$ & $\begin{array}{l}86.33 \\
(9.35)\end{array}$ & 1860.11 & 698.28 & $\begin{array}{l}12.80 \\
(3.71)\end{array}$ & $\begin{array}{l}16.70 \\
(4.20)\end{array}$ & $\begin{array}{l}13.40 \\
(3.79)\end{array}$ & 22.06 & 275.71 \\
\hline PI-5754 & 5.17 & 50.27 & 6.98 & 18.27 & 17.44 & 4.45 & 233.33 & 2.88 & $\begin{array}{c}\mathrm{G}(\mathrm{G} \\
137 \mathrm{~A})\end{array}$ & $\begin{array}{l}83.33 \\
(9.19)\end{array}$ & 1819.80 & 1230.16 & $\begin{array}{l}10.07 \\
(3.32)\end{array}$ & $\begin{array}{c}9.43 \\
(3.23)\end{array}$ & $\begin{array}{l}25.00 \\
(5.10)\end{array}$ & 15.00 & 187.54 \\
\hline PI-618894 & 5.00 & 46.67 & 5.79 & 20.00 & 25.42 & 4.50 & 275.17 & 2.54 & $\begin{array}{c}\mathrm{G}(\mathrm{G} \\
137 \mathrm{~A})\end{array}$ & $\begin{array}{l}83.33 \\
(9.19)\end{array}$ & 1801.33 & 1231.71 & $\begin{array}{c}9.73 \\
(3.27)\end{array}$ & $\begin{array}{c}3.33 \\
(2.08)\end{array}$ & $\begin{array}{c}4.00 \\
(2.23)\end{array}$ & 19.12 & 239.04 \\
\hline Poinsette & 4.87 & 62.00 & 7.77 & 24.33 & 15.58 & 4.36 & 257.87 & 3.02 & $\begin{array}{c}\mathrm{G}(\mathrm{G} \\
139 \mathrm{~B})\end{array}$ & $\begin{array}{l}94.00 \\
(9.75)\end{array}$ & 2078.27 & 1160.33 & $\begin{array}{l}11.67 \\
(3.56)\end{array}$ & $\begin{array}{c}8.90 \\
(3.14)\end{array}$ & $\begin{array}{c}9.00 \\
(3.16)\end{array}$ & 23.47 & 293.33 \\
\hline UHF-CUC-18 & 6.67 & 60.33 & 5.62 & 18.33 & 13.84 & 5.29 & 215.17 & 3.01 & $\begin{array}{l}\text { LG (G } \\
144 \mathrm{C})\end{array}$ & $\begin{array}{l}83.00 \\
(9.17)\end{array}$ & 1440.11 & 839.21 & $\begin{array}{l}10.20 \\
(3.34)\end{array}$ & $\begin{array}{l}19.20 \\
(4.49)\end{array}$ & $\begin{array}{l}27.53 \\
(5.34)\end{array}$ & 14.52 & 181.54 \\
\hline UHF-CUC-19 & 5.18 & 67.67 & 6.40 & 16.60 & 14.63 & 5.94 & 295.42 & 2.93 & $\begin{array}{l}\mathrm{LG}(\mathrm{G} \\
146 \mathrm{C})\end{array}$ & $\begin{array}{l}87.33 \\
(9.39)\end{array}$ & 1630.63 & 945.66 & $\begin{array}{l}11.73 \\
(3.57)\end{array}$ & $\begin{array}{c}7.00 \\
(2.82)\end{array}$ & $\begin{array}{l}11.40 \\
(3.52)\end{array}$ & 14.79 & 184.88 \\
\hline K-75 (Check) & 6.08 & 61.00 & 7.65 & 24.33 & 17.50 & 5.00 & 256.67 & 3.00 & $\begin{array}{l}\text { LG (G } \\
144 \text { C) }\end{array}$ & $\begin{array}{l}83.00 \\
(9.17)\end{array}$ & 1808.97 & 1199.70 & $\begin{array}{l}13.97 \\
(3.87)\end{array}$ & $\begin{array}{l}12.63 \\
(3.69)\end{array}$ & $\begin{array}{l}10.00 \\
(3.31)\end{array}$ & 19.34 & 241.71 \\
\hline Range & $\begin{array}{l}2.83- \\
9.67\end{array}$ & $\begin{array}{c}42.47- \\
67.67\end{array}$ & $\begin{array}{l}4.04- \\
8.49\end{array}$ & $\begin{array}{l}15.33- \\
29.93\end{array}$ & $\begin{array}{l}13.10- \\
25.42\end{array}$ & $\begin{array}{l}4.36- \\
6.00\end{array}$ & $\begin{array}{l}\text { 141.50- } \\
341.67\end{array}$ & $\begin{array}{l}2.53- \\
3.19\end{array}$ & & $\begin{array}{l}80.67- \\
100.00\end{array}$ & $\begin{array}{c}\text { 14410.11- } \\
2829.07\end{array}$ & $\begin{array}{l}\text { 698.28- } \\
1735.93\end{array}$ & $\begin{array}{l}6.33- \\
18.83\end{array}$ & $\begin{array}{l}3.33- \\
27.07\end{array}$ & $\begin{array}{l}4.00- \\
29.00\end{array}$ & $\begin{array}{l}11.43- \\
28.71\end{array}$ & $\begin{array}{l}142.84- \\
358.92\end{array}$ \\
\hline Mean & 5.16 & 52.02 & 6.81 & 20.80 & 16.85 & 5.02 & 268.74 & 2.88 & - & $\begin{array}{l}87.82 \\
(9.42)\end{array}$ & 1972.35 & 1218.79 & $\begin{array}{l}12.42 \\
(3.64)\end{array}$ & $\begin{array}{l}12.63 \\
(3.63)\end{array}$ & $\begin{array}{l}13.01 \\
(3.64)\end{array}$ & 20.46 & 255.81 \\
\hline $\mathrm{SE}(\mathrm{d}) \pm$ & 0.50 & 1.63 & 0.34 & 1.42 & 0.91 & 0.26 & 18.13 & 0.12 & - & 3.20 & 112.92 & 141.59 & 1.37 & 1.47 & 1.43 & 0.93 & 11.63 \\
\hline C.D.(0.05) & 0.99 & 3.26 & 0.69 & 2.85 & 1.83 & 0.51 & 36.38 & 0.24 & - & 6.41 & 226.43 & 283.94 & 2.75 & 2.95 & 2.87 & 1.87 & 23.33 \\
\hline
\end{tabular}

Where,

NNBFFF $=$ Node number bearing first female flower, NMF $=$ Number of marketable fruits per plant, FL = Fruit length $(\mathrm{cm}), \mathrm{FB}=\mathrm{Fruit}$ breadth $(\mathrm{cm}), \mathrm{AFW}=$ Average fruit weight $(\mathrm{g}), \mathrm{DMM}=$ Days to marketable maturity, $\mathrm{HD}=$ Harvest duration, $\mathrm{TSS}=$ Total soluble solids $\left({ }^{0} \mathrm{~B}\right), \mathrm{FC}=\mathrm{Fruit}$ colour, $\mathrm{LG}=\mathrm{Light}$ green, $\mathrm{G}$ $=$ Green, $\mathrm{SG}=$ Seed germination $(\%), \mathrm{SVI}-\mathrm{I}=\mathrm{Seed}$ vigor index-I, SVI-II $=$ Seed vigor index-II, SPM $=$ Severity of powdery mildew $(\%)$, SA $=$ Severity of anthracnose $(\%)$, SALS = Severity of angular leaf spot $(\%)$, YPP = Yield per plot $(\mathrm{kg})$ and $\mathrm{YPH}=$ Yield per hectare $(\mathrm{q})$

* Figures in the parenthesis are square root transformed 


\section{Cluster means}

Further, for getting the reliable conformity on the basis of cluster means, it was calculated for various horticultural traits and has been presented in the table 5. Moreover, mean performances of genotypes for different horticultural and yield traits have been presented in table 6 for getting reliable conformity about selection of parental genotypes to be used in hybridization. Genotypes of cluster III recorded lowest mean for node number bearing first female flower (3.75) along with maximum number of marketable fruits per plant (8.23), maximum average fruit weight (314.21), minimum days to marketable maturity (47.43), maximum harvest duration (28.83), highest total soluble solids (TSS) (3.04), maximum seed germination (99.42), maximum seed vigour index-I and II (2431.21, 1431.76) respectively, lowest severity of powdery mildew (9.72) and angular leaf spot (5.68). Cluster III also earned maximum yield per plot (26.93). Maximum fruit length was recorded in cluster V (21.42), while fruit breadth was highest in cluster IV (5.50). The severity of anthracnose was minimum in cluster V (7.48). Earlier workers like Sharma and Sharma (2006), Hinchinamani and Patil (2011), Punitha et al., (2012), Kumar et al., (2013) and Hasan et al., (2015) have also indicated significance of genetic divergence in cucumber.

\section{References}

Abdul-Baki, A. A. and Anderson, J. D. 1973. Vigour germinated in soyabean seed by multiple criteria. Crop Science, 13:630633.

Afangideh, U. and Uyoh, E. A. 2007. Genetic variability and correlation studies in some varieties of cucumber (Cucumis sativus L.). Jordan Journal of Agricultural Sciences, 3(4):376-384.
Akem, C. and Jovicich, E. 2011. Integrated Management of Foliar Diseases in Vegetable Crops. Department of Employment, Economic Development and Innovation, PO Box 15, Queensland 4807, Australia. 205p.

Anonymous. 1985. International rules for seed testing. Seed Science and Technology, 13:293-353.

Anonymous. 2014. Package of Practices for Vegetable Crops. Directorate of Extension Education, Dr YS Parmar University of Horticulture and Forestry, Nauni, Solan (HP). pp.61-63.

Golabadi, M., Golkar, P. and Eghtedary, A. R. 2012. Assessment of genetic variation in cucumber (Cucumis sativus L.) genotypes. European Journal of Experimental Biology, 2(5):1382-1388.

Hanchinamani, C. N. and Patil, M. G. 2011. Breeding potential of cucumber genotypes using $\mathrm{D}^{2}$ analysis. Crop Research, 41(1/3):164-167.

Hasan, R., Hossain, M. K., Nazmulalum, Bashar, A., Islam, S. and Tarafder, M. J. A. 2015. Genetic divergence in commercial cucumber (Cucumis sativus L.) genotypes. Bangladesh Journal of Botany, 44(2):201-207.

Kumar, S., Kumar, D., Kumar, R., Thakur, K. S. and Dogra, B. S. 2013. Estimation of genetic variability and divergence for fruit yield and quality traits in cucumber (Cucumis sativus L.) in North-Western Himalays. Universal Journal of Plant Science, 1(2):27-36.

Mahalanobis, P. C. 1936. On the generalized distance in statistics. Proceedings of National Academic Science, India. 2: 79-85.

McKinney, H. H. 1923. Influence of soil temperature and moisture on infection of wheat seedlings by Helminthosporium sativum. Journal of Agricultural Research, 26:195-217.

Punitha, A, Bharthi, A. and Devi, D. S. 2012. 
Studies on genetic divergence on cucumber (Cucumis sativus L.). Asian Journal of Bio Science, 7(2):169-173.

Sharma, H. R. and Sharma, D. 2006. Genetic divergence for yield and yield contributing traits in cucumber (Cucumis sativus L.). Journal of Horticultural Sciences, 1(2):141-143.

Sharma, S., Kumar, R. and Sharma, H. R. 2017. Studies on Variability, Heritability and Genetic Gain in
Cucumber (Cucumis sativus L.). Indian Journal of Ecology, 44(6):829-833.

Woltman, H. O., Bartoszewski, G., Madry, W. and Szczytt, K. N. 2009. Inheritance of resistance to angular leaf spot (Pseudomonas syringae pv. lachrymans) in cucumber and identification of molecular markers linked to resistance. Plant Pathology, 58:145-151.

\section{How to cite this article:}

Shweta Sharma, Ramesh Kumar, H.R. Sharma, Ajit Sharma and Nidhish Gautam. 2018. Divergence Studies for Different Horticultural Traits in Cucumber (Cucumis sativus L.). Int.J.Curr.Microbiol.App.Sci. 7(02): 1733-1741. doi: https://doi.org/10.20546/ijcmas.2018.702.210 